\title{
A Risk Management Approach for Software Product Line Engineering
}

\author{
Luanna Lopes Lobato \\ UFG Catalão \\ luannalobato@ufg.br
}

\author{
Thiago Jabur Bittar \\ UFG Catalão \\ thiagojabur@ufg.br
}

\begin{abstract}
Software Product Line (SPL) Engineering is a software development paradigm that fosters systematic reuse. It is focused on improving software practices, leading companies to experience benefits, such as reduced time-to-market and effort, and higher quality for the products delivered to customers. However, establishing a SPL is neither a simple nor a cheap task, and may affect several aspects of a software company. Besides, it involves a range of risks that may hinder project success. These have to be managed accordingly, so as to minimize the likelihood of project failure. Despite the importance of Risk Management (RM) for SPL Engineering, little has been published in terms of suitable and structured practices to cope with that. This present paper reports an approach for RM in SPL Engineering, named RiPLERM (Rise Product Line Engineering - Risk Management). The approach presents activities to structure RM in SPL projects, The design of the RiPLE-RM approach elaborated on results from empirical investigations, and was proposed to facilitate the management and provide significant insights that can be used to avoid and solve risks.
\end{abstract}

\section{Categories and Subject Descriptors}

D.2.2 [SOFTWARE ENGINEERING]: Design Tools and Techniques

\section{General Terms}

Management, Documentation, Design, Verification.

\section{Keywords}

Software Product Line Engineering; Risk Managemen; Project management; Software Process.

\section{INTRODUCTION}

The identification of specific and generic risks represents a critical issue for Risk Management (RM) in SPL Engineering. In a SPL, it is important to consider inherent challenges, such as: feature definition, granularity, variability, commonality, reuse, requirements definition, traceability, and other related issues that provide support to control the SPL, and avoid risk materialization [1]. Generic risks, i.e., the ones that are likely to materialize during project development, should be considered as well.

Permission to make digital or hard copies of all or part of this work for personal or classroom use is granted without fee provided that copies are not made or distributed for profit or commercial advantage and that copies bear this notice and the full citation on the first page. To copy otherwise, or republish, to post on servers or to redistribute to lists, requires prior specific permission and/or a fee.

SBSI 2015, May 26-29, 2015, Goiânia, Goiás, Brazil.

Copyright SBC 2015
It is necessary to apply a systematic method to perform RM since risk in software project is a subjective issue and demands appropriate management. An effective RM method aids problem solving, thus preventing risks propagating problems [2].

This paper reports on the RiPLE-RM (Rise Product Line Engineering), a proposed approach for Risk management in SPL Engineering. It comprises a set of activities and practices, which are used to systematize RM in SPL. Given that SPL is a rather complex development paradigm, when compared to traditional, or single-systems software development (SSD) [1], not all RM practices from SSD are completely suitable to SPL, what demands further adaptations. In this sense, this approach comes to solve the gaps identified on RM during SPL Engineering, since no methods or approaches have been reported in the literature.

As the scoping and requirement are the first SPL disciplines, in this paper is presented the proposed approach related to these disciplines. This means that considerations about scoping and requirements were carefully analyzed to develop the approach.

The remainder of this paper is structured as follows. Section 2 presents the related work. Section 3 describes underlying concepts of SPL Engineering, focusing on risk management aspects. The RiPLE-RM is detailed in Section 4 and Section 5. Section 6 presents the concluding remarks and sketch directions for further investigation.

\section{RELATED WORK}

Quilty and Cinnéide [3] present the evolution of a SSD project into a SPL scenario, highlighting the advantages identified with the SPL adoption. In their study, the high initial investment associated with SPL Engineering, in the beginning of the project, was postponed, since they performed small steps towards building an architecture for the SPL. In addition, they expanded their product into a wider market, as more complex customer requirements were added without increasing the staff number and the costs associated with the project.

They analyzed whether the benefits of SPL Engineering could be confirmed or denied during the development of the product, named as Blade, as a customization approach. Thus, they developed a set of configurable core assets that supported diverse requirements. Additionally, they could manage the risks during the transition from SSD to SPL, which presented variation between customers, due to the dynamic nature of RM.

The work of Quilty and Cinnéide [3] addresses what we consider as the closest investigation to ours, from the scarce amount of studies found in the literature. However, the similarities are limited to presenting the importance of applying RM in SPL projects, demonstrating this through results from empirical investigation. Indeed, both present a delta larger than the likely similarities. Indeed, the noticed lack of studies reporting on experiences of applying RM practices in SPL projects demonstrate a gap to bridge in this research field. 


\section{RISK MANAGEMENT IN SPL ENGINEERING}

Risk Management is an important practice to any software development activity. Adopting RM practices helps ensuring that risks are managed in an effective, efficient and coherent fashion in an organization [5].

The same holds true for SPL Engineering, especially for its particular division of responsibilities, in which the project development is split into three essential activities, as follows: (i) Core Asset Development (CAD), where the core artifacts are developed. These artifacts are entities to be further reused in (ii) Product Development (PD), by binding them according to specific products' demands; and (iii) Management (M), activity in charge of coordinating the SPL development [4]. As reuse in an inherent task in SPL Engineering, risks in a single artifact may cause problems in a range of products, that (re-)use that [1], thus RM is a driver for quality improvement.

\section{THE RIPLE-RM APPROACH}

The proposed approach is part of the RiSE Product Line Engineering (RiPLE), a framework that aggregates processes to develop SPL projects, considering the whole software life cycle, from Scoping to Testing. In this present investigation, the RiPLERM works jointly with the RiPLE-SC (SCoping) [6] and the RiPLE-RE (REquirements) processes [7]. The RiPLE-RM is founded on theoretical principles and practical actions, supported by insights collected from literature reviews on RM [9][10][11], and evidence from empirical evaluations [8][12][13][14].

The RiPLE-RM plans to assess the occurrence of risks and their impact on a SPL project. It provides a means to anticipate the risk occurrence, enabling the identification of mitigation and contingency strategies, to help minimizing the impact of risks.

Figure 1 presents an overview of the RIPLE-RM workflow, which contains a fully defined process to manage the risks. It includes the following activities: (i) Risk Communication, (ii) Risk Planning, (iii) Risk Identification, (iv) Risk Documentation, (v) Risk Assessment, (vi) Risk Analysis, (vii) Risk Treatment, and (viii) Risk Monitoring. These activities need to be systematically performed for applying RM along the project development. indication that most risks are identified during CAD [12][13]. Thus, CAD should be carefully analyzed, and the main considerations and threats should be well verified, since the assets defined in this process would be reused to assemble products. We enlisted the aspects from scoping and requirements disciplines that affect the RM activities. These are next detailed.

\subsection{SCOPING AND REQUIREMENT IN CAD}

Scoping is the first discipline of a SPL project. In such a discipline, initial decisions about the project should be set, i.e., at this point, the stakeholders decide the parts of products, features, system boundaries and domains, in which systematic reuse is economically feasible [15].

Such decisions influence the RM planning. The Risk Manager has to verify specific aspects about SPL, to analyze the benefits and risks involved in the development. Thus, some considerations about scoping development serve as inputs for the RiPLE-RM, which are herein represented by the artifact named Scoping Analysis.

Besides RM planning, scoping aspects also influence the Risk identification, assessment and monitoring. The remaining RM activities do not use this artifact as input, although they depend on these to be previously carried out. The scoping discipline directly affects the Risk Planning in a sense that decisions about the project (domain analysis, variability and commonality analysis, reuse potential assessment) could impact the planning. The Risk identification is influenced as the problems identified during scoping (domain maturity, scope size, etc.), are those that lead to a continuous analysis about the identified risks.

Scoping directly influences the Risk Assessment, as the artifact developed and the means that scoping is conducted will define the risks likelihood and impact. Finally, the Risk Monitoring is affected because some events that occur in this RM activity, can impact all the RM activities and the project during scoping development.

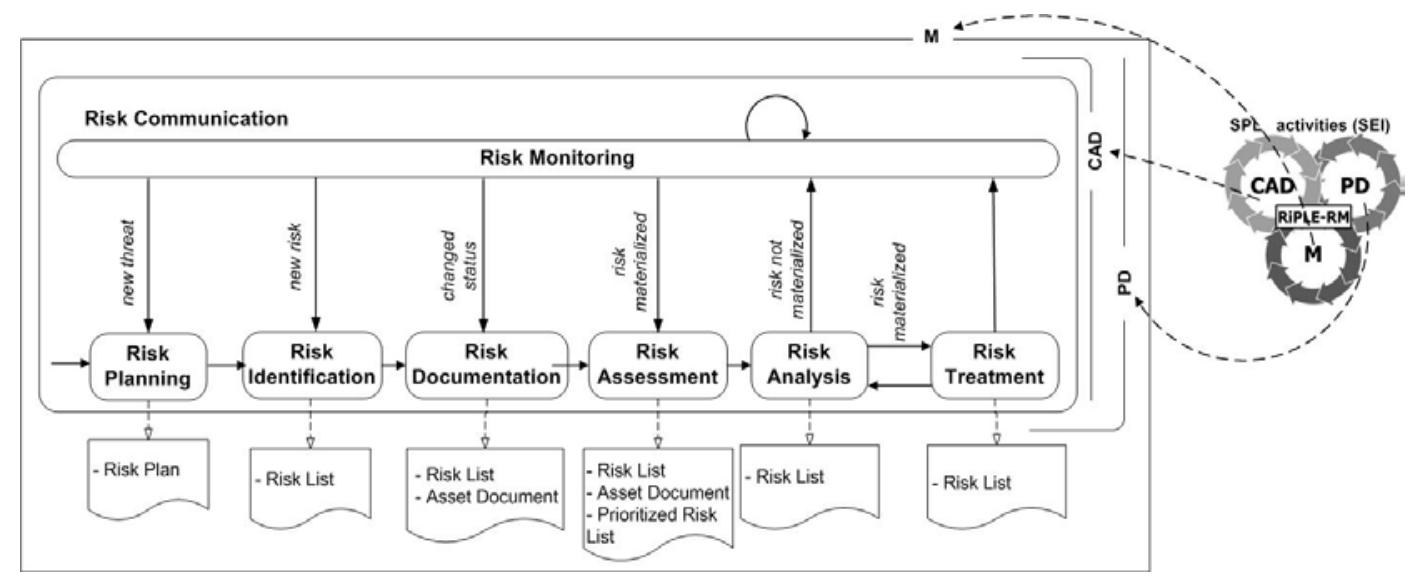

Figure 1. Overview of the RiPLE-RM workflow.

The Figure 1 shows that the RiPLE-RM considers the whole SPL lifecycle, thus including the essential activities $\mathrm{CAD}, \mathrm{PD}$ and $\mathrm{M}$. Earlier investigation points out to an
During scoping, the risks can occur in different forms, such as: there might be a lot of variability in the domain, but it might not be systematic; the reuse potential might already 
be well exploited (within the possible limits); there might be insufficient resources for exploiting the potential, etc. [16]. Thus, considering the scoping analysis artifact is a mandatory concern when performing RM during scoping.

Figure 2 presents the set of aspects to consider during scoping, and the associated roles. The scoping activities do not need to follow the strict sequence, as shown in the Figure, since these aspects are an attempt to define the activities the Risk Manager must analyze. These activities are detailed in our earlier work [8].
Domain Potential Assessment Questionnaire [16] that evaluates the domain potential. Answers for questions in this questionnaire can be classified as low, medium and high, regarding the impact of a potential risk on the project and its occurrence likelihood. Hence, it is possible to define the risk severity, and it also works as a means to classify the dangerous risks for the project, as well as to, identifying the domain areas that must be carefully analyzed.

The requirements discipline encompasses a series of important decisions, essential for the whole SPL project. It is carried out in both $\mathrm{CAD}$ and $\mathrm{PD}$, each with a particular set of tasks.

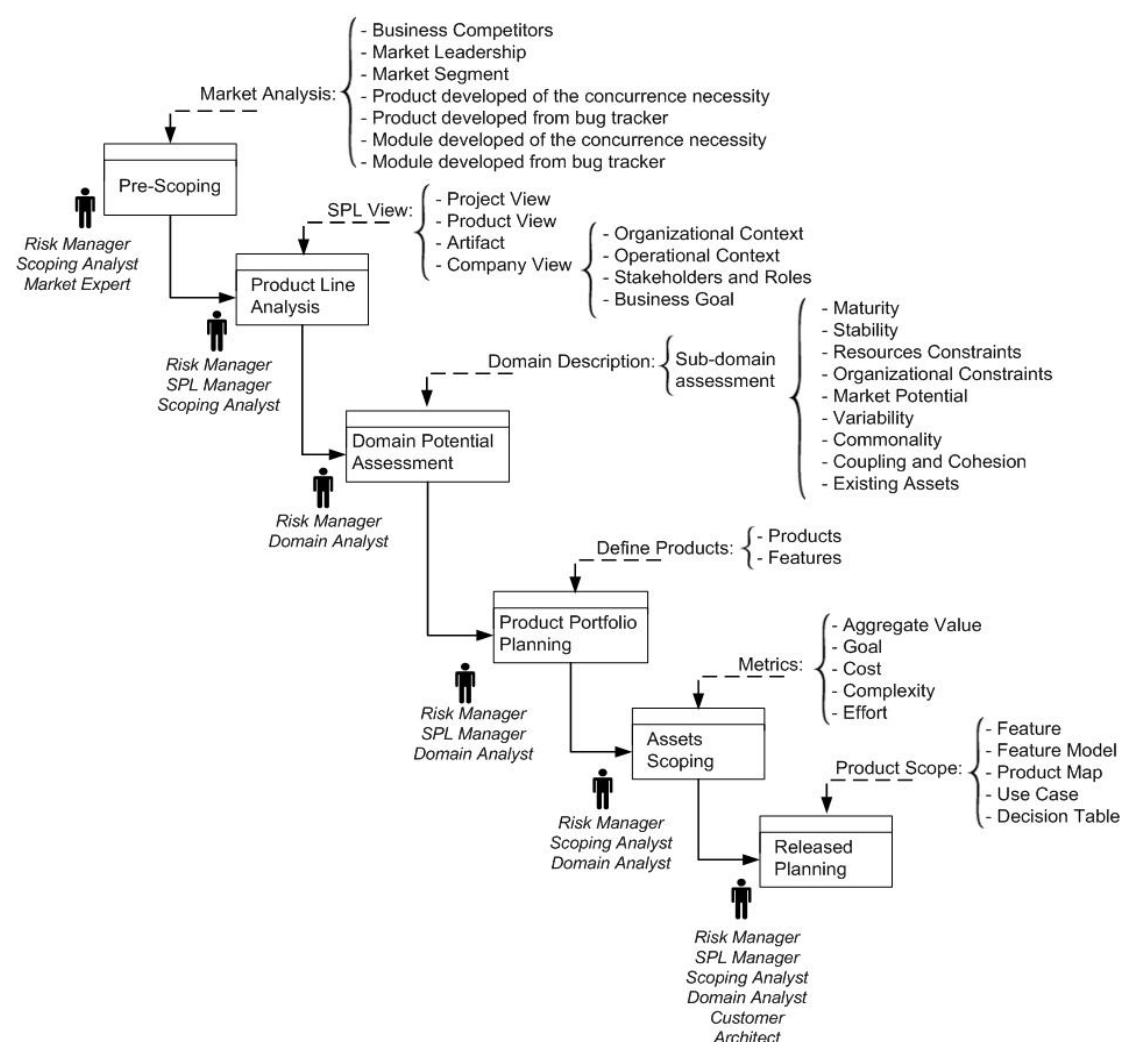

Figure 2. Scoping Analysis aspects

Among the risk management aspects to consider in the scoping definition, the Domain Potential Assessment emerges as an important artifact. In practice, the more immature the domain the more risks may be present in a project, which might harm the project development. Schmid [16] proposes a disciplined scoping approach for SPL, which assess the domain potential before institutionalizing it as a domain to the SPL. However, he does not encompass RM practices in his investigation. We took the domain potential assessment definition from such a work [16], given its importance to the RiPLE-RM.

Schmid [16] lists some specific aspects for a good scoping. They are: Viability dimensions: Maturity, Stability, Resources constraints, Organizational constraints. Benefit dimensions: Market potential - external, Market potential - internal, Commonality and Variability, Coupling and Cohesion, Existing Assets. The Risk Manager is not in charge to define which domains should be effectively developed in the SPL, but instead it is a Scoping Analyst responsibility.

In order to verify if a specific aspect can be considered a risk, the Risk Assessment task may count on a questionnaire, named as
The Requirement Analysis, aspect to consider in CAD, is performed during Risk Planning, Risk Identification, Risk Assessment, and Risk Monitoring. The Risk Manager has to analyze the Requirement Analysis aspect, from the requirements discipline, in order to figure out the likely problems that might emerge in this discipline.

After building the requirements related assets, in CAD, these are bound accordingly and assembled in products, in PD. The requirements are grouped based on the characteristics that the product should present. Alike in CAD, risks are to be managed in $\mathrm{PD}$ as well [8]

\section{RIPLE-RM ACTIVITIES}

A SPL project demands a relevant upfront effort [1], which leads to the need of considering the challenges regarding SPL adoption, and also the likely threats to the project development, so as to assess the project viability.

It is necessary to take into consideration the issues concerning to the SPL subjectivity. This encompasses the domain potential, the definition of artifacts, dependencies among the risks, variability, 
commonality, traceability among the artifacts and risks, reuse, and others.

The next sub-sections detail the RiPLE-RM activities, by describing the practices to apply risk management during SPL development.

\subsection{RISK COMMUNICATION ACTIVITY}

This is an orthogonal activity in the RiPLE-RM, since communication is a fundamental entity in RM. Its importance can be defined by means of the following statements: (i) the outputs of each activity should be communicated during the whole RM process; (ii) the stakeholders should be aware of the project during its execution, to avoid the occurrence of similar mistakes. Figure 3 presents the practices to follow in this activity.

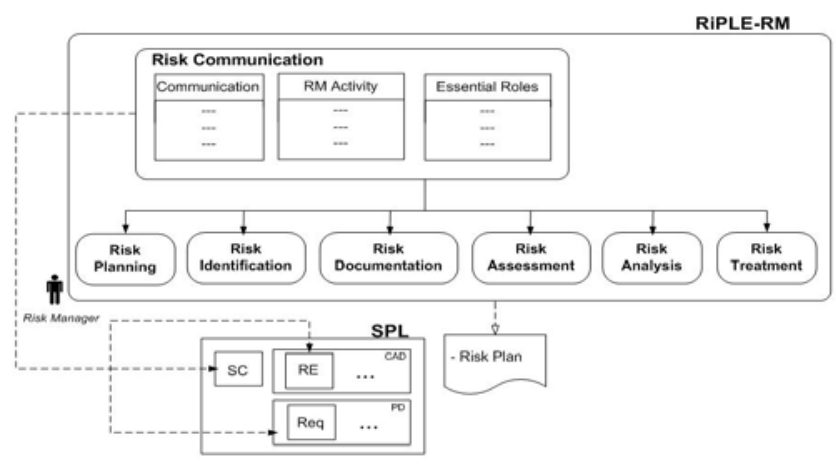

Figure 3. Risk Communication activity

Regarding Risk Communication, the Risk Manager should provide information about the identified risks in the project to both CAD and PD teams. The frequency in which the communication takes place depends on project peculiarities, being hard to make general assumptions. The practices that compose this activity are associated with: Communication Techniques: used to provide the communication between stakeholders, in order to report the findings identified during RM by the Risk Manager; RM activity: since the Communication activity is orthogonal in RM, it is important to define the activity that is being executed in order to report the outputs from the RM activity; Essential Roles: it is necessary to define the roles that are involved in the process, and the ones that should be fed with information about RM. More than one stakeholder can be associated to a same role, depending on the project size and the number of involved stakeholders (Risk Manager, SPL Manager, Scope Analyst, Requirements Analyst, Domain Analyst, Customer). Additional roles could also be considered in both scoping [6] and requirements [7]: Market Expert, Developer, Architect, Configuration Manager.

\subsection{RISK PLANNING ACTIVITY}

There are no simple processes to be followed to establish RM plans. Instead, it should rely on the judgment and expertise of the Risk Manager [2]. Plans to avoid, reduce and solve risks should be made and managed to deal with likely problems that can cause problems to the project.

The main SPL concepts are addressed in the Risk Planning activity. Figure 4 presents the aspects associated to the risk planning activity. Some artifacts from the scoping and requirements disciplines are input to the Risk Planning activity. Aiming at improving the reliability of RM, it is still necessary to analyze further issues. They are:

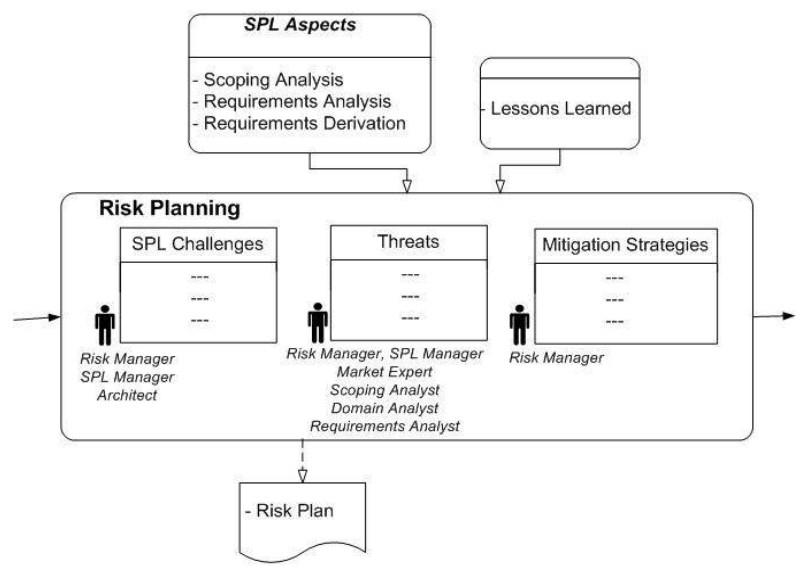

Figure 4. Risk Planning activity

SPL Aspects (input): Despite RiPLE-RM does not depend on the adoption strategies, these are analyzed to identify the threats regarding the SPL adoption [18], and thus aiding at setting realistic goals to it [19]. It is necessary to continually evaluate the progress of the SPL adoption [19], and to define strategies about how to prepare the company for the SPL adoption [20]. Some additional considerations need to be analyzed: in Scoping Analysis, the artifacts developed in the scoping discipline are analyzed. The scoping definition can present serious threats, which may harness the development activity. In this sense, threats regarding scoping development should be considered (Figure 2); in Requirements Analysis, it is necessary to consider the threats that can be faced during the development of the requirements discipline in CAD (Figure 3). In this stage the threats should be identified considering the activities necessary to develop the requirements for SPL; in Requirements Derivation, the requirements developed need to be bound to assemble products matching customers' needs. Thus, aspects of requirements derivation have to be considered as well.

Lessons Learned: The lessons learned from previous SPL projects should be considered in order to avoid that the same mistakes occur again.

SPL Challenges: The Risk Manager has to analyze these during planning, to verify the possible threats to the SPL, where underlying concepts such as Risks Traceability: related to the traceability among the artifacts. Besides, the verification should extend to the traceability between developed artifacts [21], given that a change in an artifact might affect other artifacts. A support tool is necessary, considering that keeping traceability between risks may become manually impossible, due to scale constraints; Variability/Commonality: These represent the common and variable characteristics the products from a SPL have; Variation Point/Variant: These detail what variable characteristics from a SPL could be selected to a product instance. Details about how to implement these practices are not the focus herein; Reuse: The $\mathrm{RM}$ is considered at a project level, thus as the threats or risks have been identified, the results are reused in different SPL activities.

The RiPLE-RM execution is based upon a series of reuse attributes, as follows. Heterogeneity: the Risk Manager should pay attention to heterogeneity issues, because risks are likely to occur, for example, if no pattern is followed, e.g. when documenting the project; Scalability: the number of produced artifacts increase as the project advances into different disciplines, 
then it is necessary to control such an amount of information in a specific document. The Risk Manager should work closely to the development staff in order to monitor if what is being developed is really necessary; Granularity: as several artifacts are developed in a SPL, and different teams can be involved with the project development, it is necessary to define the artifacts granularity depth. Kästner et al. [22] present the definition of correct depth about the granularity of the artifacts as a problem. This issue has to be controlled during the planning activity, considering the granularity of feature implementation; Dependencies: the Risk Manager has to search for dependencies among the artifacts, in order to identify risks that can occur with this relationship [11]; Maturity: it is necessary to verify the maturity of the artifacts that will be used in the project in order to identify problems and report this before the project start [14]; Process Maturity: the process followed in the project should be analyzed to verify its maturity, aiming at avoiding recurring problems.

Threats: after looking at specific SPL challenges, it is time to investigate, identify and define the possible threats to the project, aiming to know which problems can turn into a risk to the project. We leveraged a set of known problems that may influence the project: SPL complexity: the SPL demands good management and development practices, and the ability of the involved personnel to deal with organizational issues and architectural complexity [23]. The Risk Planning activity is the moment that such aspects must be considered to avoid possible threats to the project; Stakeholders and Organization: These are related to the people involved in the project, whose may affect the project progress [23]. It is necessary consider aspects that affect the project, in terms of human and non-human threats, and intra and inter interferences; Metrics: these can be defined to measure the cost and effort spent to apply RM in the project, as well as to define the project schedule. We applied metrics in our preceding investigation [12][13][14] to measure the time spent to manage the risks during each RM activity; Roles: these should be planned in the beginning of the project. It is important to keep all the stakeholders informed about the tasks performed and decisions taken. The SPL team should be aware about practices used by each process, in order to reaching consensus on a shared vision for the domain under development.

Mitigation Strategies: as the threats are identified, it is necessary to define some mitigation strategies during planning to avoid the risks materialization in the project. To optimize the software development and guarantee the project success, the Risk and Project Managers should previously plan the actions that can be performed to apply RM during the project development [24]. It is recommended that the planning of the RM should be developed and presented in a workshop, to the representatives' stakeholders from all the departments involved in the project, such as software, market, and sales department [20]. Findings from our preceding investigation [12][13] indicate that these workshops should occur at least once a week, with a two-hour time span. As this sounds more like a suggestion, we understand that such a decision will depend on the project size, and the Risk Manager ability as well.

\subsection{RISK IDENTIFICATION ACTIVITY}

The Risk Identification is concerned about identifying risks that occur in the project, based on the analysis performed in the Risk Planning. According to [2], the risks should not be assessed or prioritized at this stage, since the focus must be concentrated only in identifying the possible risks, without presenting details about them. Figure 5 presents the aspects to verify when performing the Risk Identification. We also indicate the involved roles.

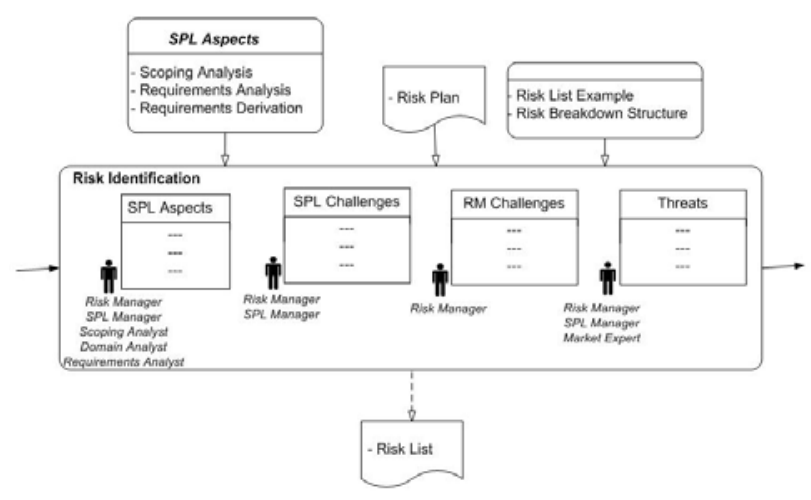

Figure 5. Risk Identification activity

Risks can emerge from several sources, as personnel organization, process and project [19]. Based on our experience in the studies developed [9] [10] [11] [12] [13] [14], we suggest some suitable techniques and methods to identify risks from such different sources: interviews, checklists, focus group and think aloud, observations, questionnaires, annotations and audio recorder, and documentation analysis. The analyzing the Risk Breakdown Structure [25] is another source of information to consider.

\subsection{RISK DOCUMENTATION ACTIVITY}

After identifying the risks, the documentation should take place, so as to enable developing a database about the actions taken, and the lessons learned. The Risk Documentation activity intends to keep the traceability among the artifacts and dependencies between the risks, e.g. to map how a risk can impact the occurrence of another risk in the project. Thus, it is essential to define a Risk Plan based on the risks that are likely to occur, and present specific techniques to avoid and mitigate their occurrence probability. It should be constantly updated based on the Risk List, which presents the risks status and categorization. The Assets Document is defined considering the traceability and dependencies. Figure 6 presents the aspects to consider, and the associated artifacts.

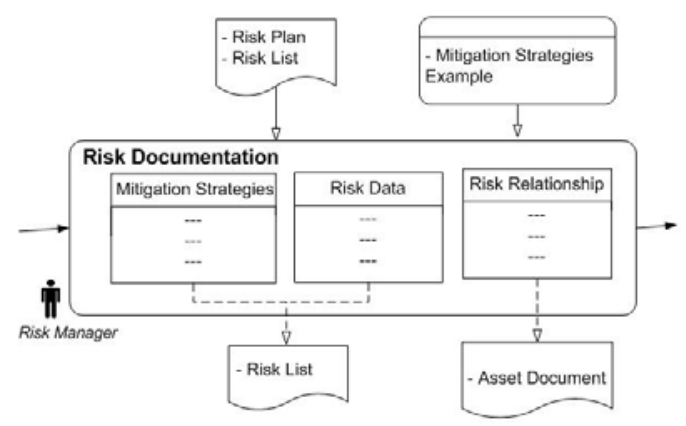

Figure 6. Risk Documentation activity

Since the Risk Documentation activity is performed according to the identified risks (in the previous RM activity), this activity is not directly influenced by the outputs from scoping and requirements disciplines.

Mitigation strategies can be developed based on the categories in that the risks are grouped. The risk classification is relevant since 
an exhaustive risk list can be created, and the documentation can be built according to the category defined.

The Risk Documentation is a continuous activity, since all insights observed during the project should be documented. Decisions need to be recognized as lessons learned, and documented to the purpose of future projects. This activity can be used to produce a risk profile, which gives significance to each risk and prepare them to assessment. The practices to conduct this RM activity are described as follows:

Mitigation Strategies: The Risk List is updated with additional information about the risks and the defined strategies. Mitigation strategies from another project should be considered as another input. In addition, company constraints and available resources should also be taken into account.

Risk Data: Details about the identified risks are documented and updated in order to define the knowledge base composed by Risk Data (risk ID, risk name, description, responsible, risk manager name, data revision and reviser name). The Risk Status can be classified as: Avoidance, Acceptance, Pendent, Reduction, Solved, Ignored, and Transferred. The risk categorization should consider: Cost, Implementation, Operational, Organizational, Schedule, Technical, and User.

Risk Relationship: It encompasses the Risk Dependencies among risks, and the Traceability among risks and artifacts. The Risk Traceability can be also used as a means to define the Risks Dependence among the risks, and the artifacts affected by them. Other RM activities, from the RiPLE-RM, also address the risks dependence and risk traceability and so these values can change. Kontio [21] highlights that whether a risk affects more than one valuable characteristic (a goal) in a project, the ranking of losses becomes non-trivial. Thus, more investments are needed to perform the RM and the traceability among the artifacts, in order to know where the risks can impact and which the problems are caused to the project.

\subsection{RISK ASSESSMENT ACTIVITY}

This activity judges the importance of each identified risk for the project. The Risk Manager defines a score of the risks and control them in a regular period of time. Companies should perform risk assessments periodically to define the likelihood and impact of the risk occurrence [3]. However, the exact period to perform the risk assessment depends on several factors, such as the project, company and, stakeholders.

It is necessary to manage the risks when introducing SPL practices. In order to define the risks severity to the project, the occurrence likelihood and impact on the project should be considered. These are based on the loss and opportunity that the occurrence of the risks represents to the project and to the company.

The artifacts developed during the RiPLE-RM are updated and developed in this activity, as Figure 7 shows. Since the SPL aspects impact in the RiPLE-RM execution during the Risk Assessment activity, it is necessary to consider the Scoping Analysis, Requirements Analysis and Requirements Definition as an input to this RM activity.

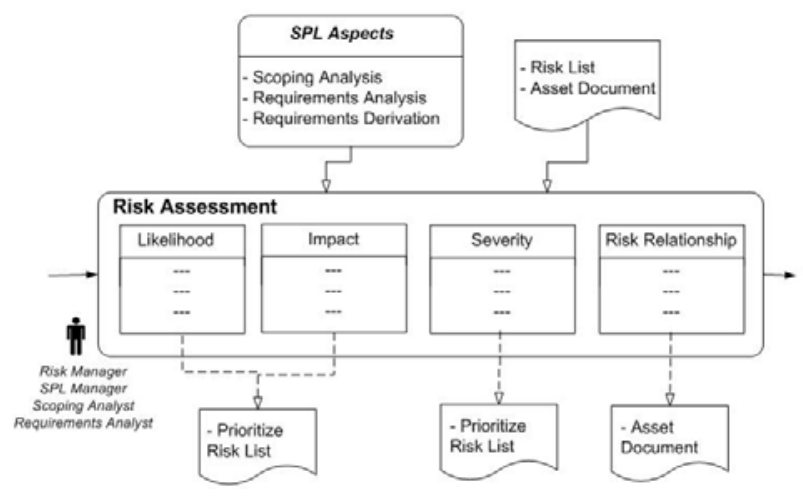

Figure 7. Risk Assessment activity

Regarding Scoping Analysis, it is necessary to consider the "Domain Potential Assessment" to figure out the risk severity (likelihood and impact) in the domain and sub-domain (immature, inadequate, low reuse potential, etc.). This is one of most serious problems that the Risk Manager needs to analyze during scoping discipline, since it can make the SPL unfeasible. In addition, it must be considered the "Product Line Analysis", "Product Portfolio Planning", "Assets Scoping" and, "Released Planning", to define the severity in terms of project execution.

It is important to highlight that the Risk Assessment is an activity directly influenced by the Risk Manager, and by the projects characteristics. Hence, it is difficult to define a single way to define the values related to the likelihood and the impact of each individual risk. In this work we assess the risks based on the likelihood and impact according to the values defined as Low (1), Medium (2) and High (3), and we suggested that the most dangerous risks to a project have to be prioritized and solved first. The Prioritize Risk List is created, where the risks are ranked based on their likelihood and impact. In our work we did not define the number of prioritized risks that should be managed.

\subsection{RISK ANALYSIS ACTIVITY}

The risks are analyzed based on the assessment performed in the previous activity. The likelihood, impact, risk status, and the SPL activities where the risk occurred are taken into account The analysis establishes a means to define the contingency strategies. Figure 8 presents the practices to conduct the Risk Analysis activity.

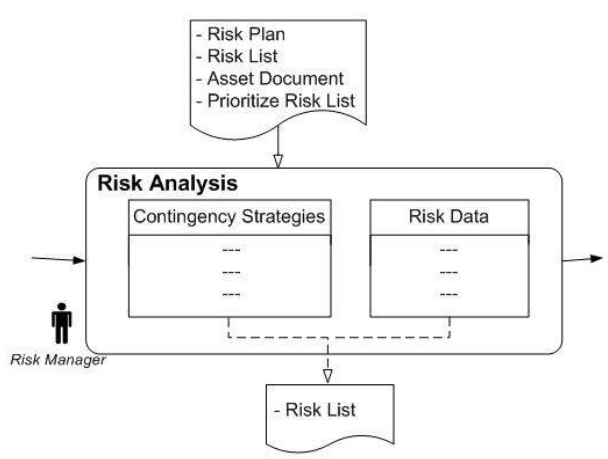

Figure 8. Risk Analysis activity

The plans to address the risk, either by avoiding or minimizing its effects on the project, should be drawn up considering the context that the project is inserted. To define the contingency strategies 
some issues should be verified, such as: organizational and project objectives, the involved stakeholders, assumptions on the project in order to anticipate possible risks, resolutions strategies, the responsible for the execution of the contingency actions, the applicability of these actions in order to guarantee that these will not impact in the occurrence of another risks, the required resources, and recovery strategies that can be used if a contingency action does not work.

Contingency Strategies: The activities for risk-reduction through the definition of contingency strategies are defined. These are strategies to reduce the impact of a risk. It is relevant to define contingency plans so that, if a risk occurs, recovery actions could be immediately taken. Once initial sets of plans are implemented the situation should be monitored.

Risk Data: These are analyzed to verify if the status has changed. They might avoid duplicated risks. It may facilitate the RM, and control the risks.

The Risk Analysis activity is used to provide the Risk Manager knowledge about the situation that the risks occurred, understand and realize the possibilities to solve them. Not only the risk as a single element, but the context in that it is inserted to define which actions can be taken to manage them. Thus, the Contingency Strategies are designed to minimize a particular risk or group of risks, i.e., to minimize the probability that a problem corresponding to the risk will occur [27].

\subsection{RISK TREATMENT ACTIVITY}

According to Quilty and Cinnéide [3], "a risk with unperformed controls can have a much higher impact than when the controls are performed". Figure 11 presents the considerations for the Risk Treatment. The artifacts developed are updated in this activity, since they can change during RM.

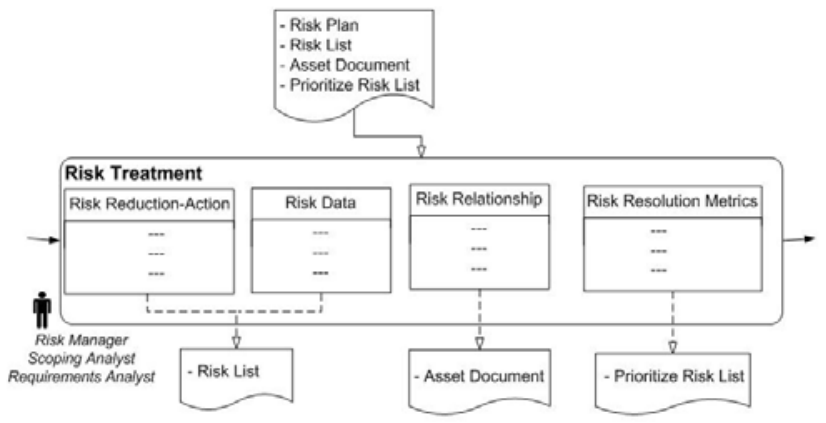

Figure 9. Risk Treatment activity

Mitigation and contingency strategies have to be applied. The risk dependencies need to be analyzed, because a risk status can change.

\subsection{RISK MONITORING ACTIVITY}

The risk may be modified as new risks information emerges. Hence, the RM should be a continually monitored process where the risks and their status are periodically verified. In addition, should be identified and monitored mainly the "top ten" risks, which are the risks considered the most dangerous to the project success. However, we understand that the right number of risks to monitor must depend on every project. Figure 10 presents some actions that can be taken during the Risk Monitoring.

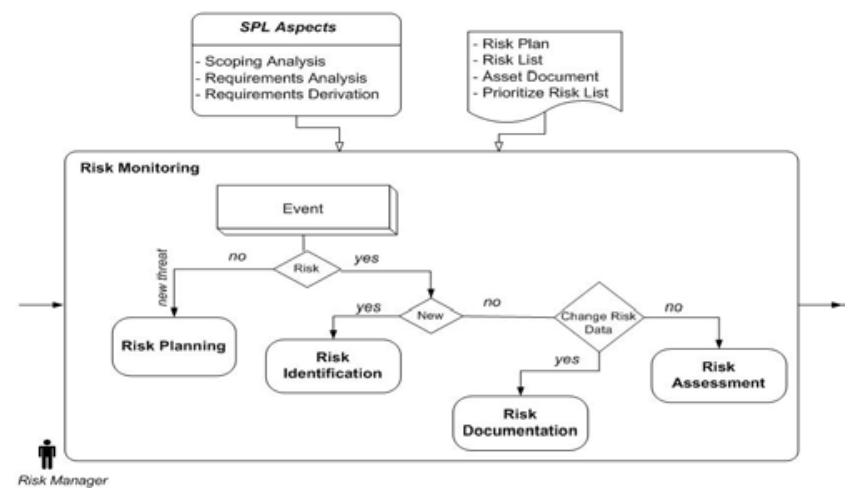

Figure 10. Risk Monitoring activity

It is necessary to verify if the event is a risk or if it was only identified as a threat to the project. The threats are those problems that are not a risk to the project, and the risk is a threat that becomes real to the project. If it is a threat, the Risk Planning activity is performed, otherwise it is necessary to analyze if this risk have already been identified in the project. Thus, if the event is new, the Risk Identification is performed. If not, it is verified which change makes the event happen. If there are changes related to risks data, the Risk Documentation is performed, and if the changes are related to the likelihood and impact, the Risk Assessment activity will be performed to assess the risks and define again the risk severity, as well as the risk ranking.

\section{CONCLUSIONS}

The RiPLE-RM was proposed based on data gathered from several sources [8]. An initial approach version was applied and verified in a series of case studies [12] [13], to leverage improvements opportunities. Besides, the proposed RiPLE-RM also considered feedbacks from experts [12], which aided at identifying the benefits and drawbacks of the RiPLE-RM.

In addition, a controlled experiment was performed in order to assess the benefits and drawbacks with the use of the RiPLE-RM [12]. Since external interventions are involved with the application of experiments in a real scenario, we agree with the claim that rigorous experiment demands much time to be performed, and, sometimes, additional investments. Besides, it is tough to find companies willing to introduce academic proposals to their development process. In case of our process evaluation, we would demand companies to start a new SPL project and agree that the RiPLE-RM could be validated during the project development, which satisfies a hard constraint to cope with. Hence, such constraints lead us to perform a controlled experiment in the academic context, where Master and Phd students acted as participants. We understand that such scenario may not enable us to generalize our findings to a diversity of scenarios. However, this study was an initial point towards validating our proposal, and may serve to establish baseline values for future studies. Thus, the participants were divided into two groups, where the expertise was analyzed to define the groups, based on data gathered with their experience in RM and SPL. Despite the groups' definition, in which the first group should apply RM with support of the RiPLE-RM, as opposed to the second one, each member of every group had to analyze the $\mathrm{RM}$ in an individual fashion. Thus, the results reported in this work were verified based on the group defined and in the subjects' answers as well. 
We observed that the subjects from both groups presented different risks. This was already expected because RM is a subjective task. Different professionals may have different ideas about what is and what is not a risk considering the same scenario. Mitigation and contingency strategies also vary. Thus, the activity of managing risks depends on the felling of the Risk Manager.

It was verified that the process to perform RM during projects development can be slow and boring if no support is given to the Risk Manager, related to aid about what should be done and which RM activities to be executed. Thus, the RiPLE-RM was proposed in order to provide guidelines to be applied during RM in SPL project, in scoping and requirement disciplines. Regarding the lessons learned, the RM should be performed at least once a week, since its delay can make risks more dangerous to the project success. The details about these studies were not detailed due to page limitation. For more details please see [12].

As conclusion, we verified that RM is still an immature field to SPL Engineering, mainly in terms of RM methods. In this investigation, we try to bridge such a gap by proposing the RiPLE-RM, a systematic approach to cope with RM in SPL. We describe and detail each activity and task required to handle risks in a SPL project and discussed relevant aspects that can impact the SPL projects and thus, may represent risk potential.

Indeed, despite the systematic approach presented, we understand that RM still depends on the competence and expertise of the involved stakeholders. Thus, RM is subjective when compared to the execution of process where the results depends on controlled variables much more than the performance of the people involved in the process. A limitation in this investigation refers to the development of such an assessment model for RM in SPL. However, as this field is still not mature, we believe that our findings may provide the community with a good starting point for developing a model in the future.

Future investigation includes extending the RiPLE-RM to the whole SPL lifecycle, and also to consider risks emerging from organizational aspects, besides the technical point of view of the current investigation.

\section{REFERENCES}

[1] Clements, P. and Northrop, L. Software Product Lines: Practices and Patterns. Addison-Wesley, Boston, MA, USA. 2001.

[2] Sommerville, I. Software engineering (8th ed.). Redwood City, CA, USA: Addison Wesley Longman Publishing Co., Inc., 2007.

[3] Quilty, G. and Cinnéide, M. Ó. Experiences with Software Product Line Development in Risk Management Software. Software Product Line Conference. Munich, august 2011.

[4] Northrop, L. M. and Clements, P. C. A framework for software product line practice, version 5.0. Technical report, Software Engineering Institute (SEI). 2007.

[5] ISO 31000. Risk management - Principles and guidelines. First edition.

[6] Moraes, M. B. S., Almeida, E. S. and Meira, S. L. A scoping process for software product lines. In 23rd Intl. Conference on Software Engineering and Knowledge Engineering, Miami, USA, 2011.

[7] Neiva, D. F. S., de Almeida, F. C., de Almeida, E. S. and Meira, S. R. L. A requirements engineering process for software product lines.
In 11th IEEE International Conference on Information Reuse and Integration (IRI), Las Vegas, U.S. 2010.

[8] Lobato, L. L. An approach for Risk Management in Software Product Lines. Ph.D. Thesis. Federal University of Pernambuco, Recife - Brazil, 382p. 2012.

[9] Lobato, L. L., Silveira Neto, P. A. M., Machado, I. C., Almeida, E. S. and Meira, S. R. L. Risk Management in Software Product Lines: A Mapping Study. In: International Journal of Software Engineering and Knowledge Engineering. Vol. 23, No. 3 (2013) 1-36.

[10] Lobato, L. L., Silveira Neto, P. A. M., Machado, I. C., Almeida, E. S. and Meira, S. R. L. An Study on Risk Management for Software Engineering. In: 16th International Conference on Evaluation \& Assessment in Software Engineering, Ciudad Real, Spain. 2012.

[11] Lobato, L. L., Machado, I., Neto, P. A. M. S., Almeida, E. S. and Meira, S. R. L. (2012). Synthesizing Evidence on RM to Software Engineering: A Narrative Synthesis of the Scoping Studies. In: International Conference on Software Engineering and Knowledge Engineering, San Francisco Bay, USA. 2012.

[12] Lobato, L. L., Machado, I. C., Silveira Neto, P. A. M., Almeida, E. S., Meira, Silvio Romero de Lemos . On the investigation of Risk Management Practices in Software Product Lines. In: Simpósio Brasileiro de Qualidade de Software (SBQS), Fortaleza. 2012.

[13] Lobato, L. L., Silveira Neto, P. A. M., Machado, I. C., Almeida, E. S., Meira, Silvio Romero de Lemos. Risk Management in Software Product Lines: An Industrial Case Study. In: International Conference on Software and System Process (ICSSP), Zurich, Switzerland. 2012.

[14] Lobato, L. L., Machado, I., Neto, P. A. M. S., Almeida, E. S. and Meira, S. R. L. Evidence from RM in SPL projects development: a Cross Case Analysis. In: Simpósio Brasileiro de Componentes, Arquitetura e Reutilização de Software, 2012, Natal, Brazil.

[15] John, I. and Eisenbarth, M. A decade of scoping: a survey. In: 13th International Software Product Line Conference. Carnegie Mellon University, Pittsburgh, PA, USA. 2009.

[16] Schmid, K. An assessment approach to analyzing benefits and risks of product lines. In: 25th International Computer Software and Applications Conference, COMPSAC, pages 525-530, Washington, DC, USA. IEEE Computer Society. 2001.

[17] Alves, V., Niu, N., Alves, C., and Valença, G. Requirements engineering for software product lines: A systematic literature review. Information Software Technology, 52(8), 806-820. 2010.

[18] Bastos, J. F., Silveira Neto, P. A. S., de Almeida, E. S., and de Meira, S. R. L. Adopting software product lines: A systematic mapping study. In: 15th International Conference on Evaluation and Assessment in Software Engineering, Durham, England. 2011.

[19] Bandinelli, S. and Mendieta, G. S. Domain Potential Analysis: Calling the Attention on Business Issues of Product-Lines. In: International Workshop on Software Architectures for Product Families (IW-SAPF-3), Frank van der Linden (Ed.). SpringerVerlag, London, UK, 76-81. 2000.

[20] Sagarduy, G., Bandinelli, S. and Lerchundi, R. Product-line Analysis: Do we go ahead? In: Proceedings of the international Workshop on Software Product Lines: Economics, Architecture and Implications. 2000.

[21] Kontio, J. Software Engineering Risk Management: A Method, Improvement Framework, and Empirical Evaluation. Ph.D. Thesis, Department of Computer Science and Engineering, Hensinki University of Technology, Finland. 2001.

[22] Kästner, C. Apel, S. and Kuhlemann, M. Granularity in software product lines. Proc. of the 30th international conference on Software engineering (ICSE). ACM, New York, NY, USA, 311-320. 2008. 